\title{
A New Method to Create a Virtual Third Antenna from a Two-Channel SAR-GMTI System
}

\author{
Martina Gabele \\ German Aerospace Center (DLR) - Wessling \\ Satellite SAR Systems Section \\ Muenchner Str. 20, 82234 Wessling, Germany \\ E-mail: Martina.Gabele@dlr.de
}

\author{
Ishuwa Sikaneta \\ Defence R\&D Canada - Ottawa \\ Radar Systems Section \\ 3701 Carling Ave., Ottawa, ON, Canada, K1A 0Z4 \\ E-mail: ishuwa.sikaneta@drdc-rddc.gc.ca
}

\begin{abstract}
Two-channel SAR-GMTI systems are suboptimal for moving target motion parameter estimation. Indeed, the ATI phase estimate of the across-track velocity component for a moving target is biased to lower values depending on the target signal to clutter ratio and the target across-track velocity. Additional antenna diversity can introduce additional degrees of freedom that can eliminate the bias problem. Aperture switching is an accepted method to virtually increase the number of channels without adding new hardware. One such mode is the RADARSAT-2 Toggle mode [1]. This paper proposes a new processing method to create a similar effective phase center configuration as the RADARSAT- 2 Toggle mode from already recorded two-channel SAR data. This is achieved by delaying and combining the recorded two-channel measurements. The combination operation manifests not only a third phase center halfway between the phase centers of the two-channel system, but also a different antenna length of the virtual third antenna which requires a modification of the DPCA-ATI processing algorithm. The DPCA-ATI performance of the new mode is assessed and compared to ATI from the original two-channel mode.
\end{abstract}

\section{INTRODUCTION}

In a synthetic aperture radar (SAR) image moving target signals appear at a shifted azimuth position and hence, interfere with the clutter signal at this position. The azimuth shift is dependent on the target across-track velocity component. The challenge in target motion parameter estimation is to first suppress the clutter signal at the same azimuth position and then estimate the target across-track velocity of the moving target. Two-channel SAR ground moving target indication (GMTI) systems are suboptimal, because in a linear processor there exists only one degree of freedom that can be used either for clutter cancellation or for parameter estimation, but not both. For example, clutter increasingly biases the two-channel along-track interferometry (ATI) phase estimate as the signalto-clutter ratio (SCR) decreases [4]. Additional antenna diversity (three or more antennas) can introduce additional degrees of freedom which allow combined clutter cancellation and parameter estimation. In the three antenna case, one proposed method combines displaced phase center antenna (DPCA) for clutter suppression with along-track interferometry (ATI) for across-track velocity estimation.

Aperture switching is an accepted method to virtually increase the number of channels without adding new hardware. The basic idea is to generate additional antenna phase centers by alternating, from pulse to pulse, the transmit and/or receive antenna configurations. One such mode is the RADARSAT-2 Toggle mode [1] which alternates between fore and aft antenna on transmit while receiving with both antennas. This method generates two phase centers at the real antenna phase center positions of fore and aft antenna as well as a phase center halfway between the two antennas.

This paper proposes a new processing method to create a similar effective phase center configuration as the RADARSAT-2 Toggle mode from already recorded twochannel SAR data. This is achieved by delaying and summing the recorded two-channel measurements. The combination operation manifests a third phase center halfway between the phase centers of the two-channel system, but it introduces also a different antenna pattern weighting of the medium channel signals due to the double length of the virtual third antenna. This requires a modification of the DPCA-ATI processing algorithm.

The outline of the paper is as follows: In section II the moving target signals of fore and aft channel are derived as well as the signals of the clutter at the azimuth displaced position, which is from now on called the corresponding clutter signal. Then the sum channel signals of moving target and corresponding clutter are derived. Section III analyzes the impact of the SAR processing on the real data signals as well as the sum channel signals. Finally, in section IV the moving target parameter estimation using a DPCA-ATI combination is compared to estimation using classical ATI for the two-channel data.

\section{SAR RAW DATA Signals}

Let us assume without loss of generality that a moving target at azimuth position $x_{0}=0 \mathrm{~m}$ relative to the zero azimuth position of the aft channel and slant range position $y_{0}$ is moving with along-track velocity $v_{x}$ and slant range acrosstrack velocity $v_{y}$ while the platform is moving with velocity $v_{p}$. The fore channel is displaced in the along-track direction by $d$ ahead of the aft channel. The range histories $R\left(t, x_{d}\right)$ versus time $t$ of the SAR raw data are then:

$$
R\left(t, x_{d}\right)=\sqrt{\left[x_{d} / 2+\left(v_{p}-v_{x}\right) t\right]^{2}+\left[y_{0}+v_{y} t\right]^{2}},
$$


where $x_{d}=0$ for the aft channel, and $x_{d}=d$ for the fore channel. A second order Taylor approximation around $t=0 \mathrm{~s}$ yields:

$$
R\left(t, x_{d}\right)=y_{0}+v_{y} t+\frac{v_{r e l}^{2}}{2 y_{0}} t^{2}+\left(v_{p}-v_{x}\right) \frac{x_{d}}{2 y_{0}} t,
$$

where $v_{r e l}=\sqrt{\left(v_{p}-v_{x}\right)^{2}+v_{y}^{2}}$. Quadratic completion of the range histories and the fact that the SAR signal is $s(t)=$ $w(t) \cdot \exp (-j 2 \beta R(t))$ results in the following equation for the moving target SAR raw data signal $s\left(t, x_{d}\right)$ :

$$
\begin{gathered}
s\left(t, x_{d}\right)=w(t) \cdot \exp \left(j \frac{\beta x_{d} v_{y}}{v_{e}}\right) \cdot \\
\cdot \exp \left(-j \beta\left[\frac{v_{r e l}^{2}}{y_{0}}\left(t+\frac{x_{d}}{2 v_{e}}-\Delta t\right)^{2}+2 y_{0}-\frac{v_{y}^{2} y_{0}}{v_{r e l}^{2}}\right]\right),
\end{gathered}
$$

where $w(t)$ is an arbitrary antenna pattern weighting, $\beta$ is the wavenumber, $\Delta t=-y_{0} v_{y} / v_{r e l}^{2}$, and $v_{e}=\left(v_{p}-v_{x}\right)+$ $v_{y}^{2} /\left(v_{p}-v_{x}\right)$. The SAR raw data signal of the corresponding clutter $c\left(t, x_{d}\right)$ is:

$$
\begin{gathered}
c\left(t, x_{d}\right)=w(t-\Delta t) \cdot \\
\cdot \exp \left(-j \beta\left[\frac{v_{p}^{2}}{y_{0}}\left(t+\frac{x_{d}}{2 v_{p}}-\Delta t\right)^{2}+2 y_{0}\right]\right) .
\end{gathered}
$$

Taking into account that $v_{p} \approx v_{r e l}$, (3) and (4) show that the quadratic part of the phase history of a moving target signal is equal to the quadratic part of the phase history of the corresponding clutter signal. The moving target signals differ in a constant phase factor $\exp \left(j \beta d v_{y} / v_{e}\right)$ between the fore and aft channels.

By using the Doppler frequency representation of stationary targets, (see [8]) and the time domain representation of (3) along with some elementary Fourier Transform properties, the moving target SAR raw data signal in the Doppler domain can be written as

$$
\begin{gathered}
S\left(f, x_{d}\right)=A(f-\Delta f) \cdot \exp \left(j 2 \pi t_{0} f\right) \cdot \exp \left(j \frac{\beta x_{d} v_{y}}{v_{e}}\right) . \\
\cdot \exp \left(-j 2 \beta y_{0} \sqrt{1-\left(\frac{\lambda}{2 v_{r e l}}(f-\Delta f)\right)^{2}}\right)
\end{gathered}
$$

where $\mathcal{F}\{w(t)\}=W\left(-\left(y_{0} \lambda(f-\Delta f)\right) /\left(2 v_{r e l}^{2}\right)\right)=A(f-$ $\Delta f), \Delta f=-2 v_{y} / \lambda$ is the Doppler frequency shift due to the target across-track motion $v_{y}$, and $t_{0}=x_{d} /\left(2 v_{e}\right)$. The corresponding clutter signal $C\left(f, x_{d}\right)$ from (4) becomes

$$
\begin{aligned}
C\left(f, x_{d}\right) & =A(f) \cdot \exp \left(-j 2 \beta y_{0} \sqrt{1-\left(\frac{\lambda f}{2 v_{p}}\right)^{2}}\right) . \\
& \cdot \exp \left(j 2 \pi t_{0} f\right) \cdot \exp (-j 2 \pi \Delta t f),
\end{aligned}
$$

\section{SAR PRocessing of REAL AND SUM ChANNEL SIGNALS}

One can write the frequency domain representation of the signals of the two-channel system as a vector

$$
\vec{S}(f)=\exp \left(-j 2 \beta y_{0} \sqrt{1-\left[\frac{\lambda}{2 v_{r e l}}(f-\Delta f)\right]^{2}}\right) .
$$

$\cdot\left[\begin{array}{c}A_{1}(f-\Delta f) \\ A_{2}(f-\Delta f)\end{array}\right] \otimes\left[\begin{array}{c}1 \\ \exp \left\{j 2 \pi t_{0} f\right\}\end{array}\right] \otimes\left[\begin{array}{c}1 \\ \exp \left\{j \frac{\beta d v_{y}}{v_{e}}\right\}\end{array}\right]$,

where $\otimes$ denotes element-wise multiplication (or the Schur product). For stationary clutter, $v_{y}=0$. The SAR processing filter with candidate across-track velocity $\hat{v}_{y}$, and $\Delta \hat{f}=$ $-2 \hat{v}_{y} / \lambda$ can be written as

$$
H_{\mathrm{SAR}}(f)=\exp \left\{-\frac{j \beta y_{0} \lambda^{2} f^{2}}{4 v_{r e l}^{2}}+j 2 \pi f \frac{y_{0} \lambda}{2 v_{r e l}^{2}} \Delta \hat{f}\right\} .
$$

In order to generate the sum channel, we construct the matrix

$$
\mathbf{M}_{l i n}=\left[\begin{array}{cc}
A_{2}(f-\Delta \hat{f}) & 0 \\
\frac{1}{2} A_{2}(f-\Delta \hat{f}) & \frac{1}{2} A_{1}(f-\Delta \hat{f}) \\
0 & A_{1}(f-\Delta \hat{f})
\end{array}\right] .
$$

The calibration of the fore and aft channel to the sum channel can be expressed as follows:

$$
\mathbf{M}_{\cos }=\left[\begin{array}{ccc}
\cos \left[C_{1}(f-\Delta \hat{f})\right] & 0 & 0 \\
0 & 1 & 0 \\
0 & 0 & \cos \left[C_{1}(f-\Delta \hat{f})\right]
\end{array}\right] .
$$

with $C_{1}=\pi d /\left(2 v_{e}\right)$. The calibrated signal becomes

$$
\mathbf{M}_{c o s} \mathbf{M}_{l i n} H_{S A R}(f) \vec{S}(f)
$$

$$
\begin{gathered}
=\exp \left\{-j 2 \beta\left[1-\frac{v_{y}^{2}}{2 v_{r e l}^{2}}\right]\right\} \exp \left\{j 2 \pi f \frac{y_{0} \lambda}{2 v_{r e l}^{2}}(\Delta f-\Delta \hat{f})\right\} \\
\cdot\left[\begin{array}{c}
R(f, \Delta f, \Delta \hat{f}) \cos \left[C_{1}(f-\Delta \hat{f})\right] \\
\frac{R(f, \Delta f, \Delta \hat{f}) \exp \left[-j C_{1}(f-\Delta f)\right]}{2}+\frac{R(f, \Delta \hat{f}, \Delta f) \exp \left[j C_{1}(f-\Delta f)\right]}{2} \\
R(f, \Delta \hat{f}, \Delta f) \cos \left[C_{1}(f-\Delta \hat{f})\right]
\end{array}\right] \\
\otimes\left[\begin{array}{c}
1 \\
\exp \left(j \pi f t_{0}\right) \\
\exp \left(j 2 \pi f t_{0}\right)
\end{array}\right] \otimes\left[\begin{array}{c}
1 \\
\exp \left(j \frac{\beta d v_{y}}{2 v_{e}}\right) \\
\exp \left(j \frac{\beta d v_{y}}{v_{e}}\right)
\end{array}\right],
\end{gathered}
$$

where $R(f, \Delta f, \Delta \hat{f})=A_{1}(f-\Delta f) A_{2}(f-\Delta \hat{f})$. Coregistration can be achieved pre-multiplying with the matrix 


$$
\mathbf{M}_{\mathrm{reg}}=\left[\begin{array}{ccc}
1 & 0 & 0 \\
0 & \exp \left(-j \pi f t_{0}\right) & 0 \\
0 & 0 & \exp \left(-j 2 \pi f t_{0}\right)
\end{array}\right] .
$$

In the case of identical antenna patterns, $R(f, \Delta f, \Delta \hat{f})=$ $R(f, \Delta \hat{f}, \Delta f)$, and one can write

$$
\begin{gathered}
\mathbf{M}_{\mathrm{reg}} \mathbf{M}_{\mathrm{cos}} \mathbf{M}_{\mathrm{lin}} H_{\mathrm{SAR}}(f) \vec{S}(f) \\
=\exp \left(-j 2 \beta\left[1-\frac{v_{y}^{2}}{2 v_{r e l}^{2}}\right]\right) \exp \left(j 2 \pi f \frac{y_{0} \lambda}{2 v_{r e l}^{2}}(\Delta f-\Delta \hat{f})\right) \\
\cdot\left[\begin{array}{c}
R(f, \Delta f, \Delta \hat{f}) \cos \left[C_{1}(f-\Delta \hat{f})\right] \\
R(f, \Delta f, \Delta \hat{f}) \cos \left[C_{1}(f-\Delta f)\right] \\
R(f, \Delta f, \Delta \hat{f}) \cos \left[C_{1}(f-\Delta \hat{f})\right]
\end{array}\right] \otimes\left[\begin{array}{c}
1 \\
\exp \left(j \frac{\beta d v_{y}}{2 v_{e}}\right) \\
\exp \left(j \frac{\beta d v_{y}}{v_{e}}\right)
\end{array}\right] .
\end{gathered}
$$

By introducing the frequency average and difference variables $f_{1}=(\Delta f+\Delta \hat{f}) / 2$, and $f_{2}=(\Delta f-\Delta \hat{f}) / 2$, one can write the expression for the Doppler representation of the compressed SAR signals as

$$
\begin{gathered}
\mathbf{M}_{\mathrm{reg}} \mathbf{M}_{c o s} \mathbf{M}_{\mathrm{lin}} H_{\mathrm{SAR}}(f) \vec{S}(f)=\left[\begin{array}{c}
1 \\
\exp \left(j \frac{\beta d v_{y}}{2 v_{e}}\right) \\
\exp \left(j \frac{\beta d v_{y}}{v_{e}}\right)
\end{array}\right] \\
\otimes\left[\begin{array}{l}
R\left(f-f_{1}, f_{2},-f_{2}\right) \cos \left[C_{1}\left(f-f_{1}+f_{2}\right)\right] \\
R\left(f-f_{1}, f_{2},-f_{2}\right) \cos \left[C_{1}\left(f_{1}-f+f_{2}\right)\right] \\
R\left(f-f_{1}, f_{2},-f_{2}\right) \cos \left[C_{1}\left(f-f_{1}+f_{2}\right)\right]
\end{array}\right] \\
\cdot \exp \left(-j 2 \beta\left[1-\frac{v_{y}^{2}}{2 v_{r e l}^{2}}\right]\right) \exp \left(j 2 \pi f \frac{y_{0} \lambda}{v_{r e l}^{2}} f_{2}\right) .
\end{gathered}
$$

When the antenna pattern $A(f)$ has either even or odd symmetry $R\left(f-f_{1}, f_{2},-f_{2}\right)=R\left(f_{1}-f, f_{2},-f_{2}\right)$, one can see that the envelope of the middle antenna is the reflection of the envelope of the fore and aft antennas. Let us denote the frequency envelope of the fore antenna as $P(f)=$ $R\left(f, f_{2},-f_{2}\right) \cos \left[C_{1}\left(f+f_{2}\right)\right]$. One can now express (14) as

$$
\mathbf{M}_{r e g} \mathbf{M}_{\mathrm{cos}} \mathbf{M}_{l i n} H_{S A R}(f) \vec{S}(f)=C_{2} \exp \left(j 2 \pi f C_{3}\right) .
$$

$$
\cdot\left[\begin{array}{l}
P\left(f-f_{1}\right) \\
P\left(f_{1}-f\right) \\
P\left(f-f_{1}\right)
\end{array}\right] \otimes\left[\begin{array}{c}
1 \\
\exp \left(j \frac{\beta d v_{y}}{2 v_{e}}\right) \\
\exp \left(j \frac{\beta d v_{y}}{v_{e}}\right)
\end{array}\right]
$$

where $C_{2}=\exp \left(-j 2 \beta\left[1-v_{y}^{2} /\left(2 v_{r e l}^{2}\right)\right]\right)$, and $C_{3}=$ $f_{2} y_{0} \lambda / v_{r e l}^{2}$. By using the Fourier Transform properties of frequency shift, frequency modulation, and reflection the time domain representation of the compressed and coregistered SAR signals (15) can be written as

$$
\vec{s}(t)=\mathcal{F}^{-1}\left\{\mathbf{M}_{r e g} \mathbf{M}_{c o s} \mathbf{M}_{\text {lin }} H_{S A R}(f) \vec{S}(f)\right\}
$$

$$
=C_{4}(t)\left[\begin{array}{c}
p\left(t-\left(v_{y}-\hat{v}_{y}\right) \frac{y_{0}}{v_{r e l}^{2}}\right) \\
p\left(-t+\left(v_{y}-\hat{v}_{y}\right) \frac{y_{0}}{v_{r e l}^{2}}\right) \\
p\left(t-\left(v_{y}-\hat{v}_{y}\right) \frac{y_{0}}{v_{r e l}^{2}}\right)
\end{array}\right]\left[\begin{array}{c}
1 \\
\exp \left(j \frac{\beta d v_{y}}{2 v_{e}}\right) \\
\exp \left(j \frac{\beta d v_{y}}{v_{e}}\right)
\end{array}\right],
$$

where $\mathcal{F}^{-1}\{P(t)\}=p(t)$, and $C_{4}(t)=C_{2} \exp (j 2 \pi(t+$ $\left.\left.C_{3}\right) f_{1}\right)$. The above expression shows that the compressed signals of all channels, including the synthetic channel, have a common phase for stationary targets. When a moving target has been imaged, each channel manifests a phase proportional to the target radial speed $v_{y}$, with the middle channel phase half that of the aft channel phase. The compressed envelope functions of all antennas are centered on the same point, $\left(v_{y}-\hat{v}_{y}\right) y_{0} v_{r e l}^{2}$, however, the middle channel envelope is the reflection of the fore and aft envelope. When the SAR data are properly focussed one expects $p(t)$ to be narrow to achieve the high resolution of a SAR. However, some phase spreading of the DPCA-ATI signal may occur due to a non point-like impulse respone, especially when an incorrect Doppler rate is used to process the moving target.

In figure 1 the absolute values of the SAR raw data signals are shown before (top) and after antenna pattern calibration (bottom), where the antenna pattern calibration is adapted to the moving target signal. Because the antenna patterns of moving target and corresponding clutter are shifted against each other in Doppler frequency, the calibration which is required to equalize the corresponding clutter fore and aft channel signal patterns to the medium channel is different from the calibration that is required to equalize the moving target signal patterns.
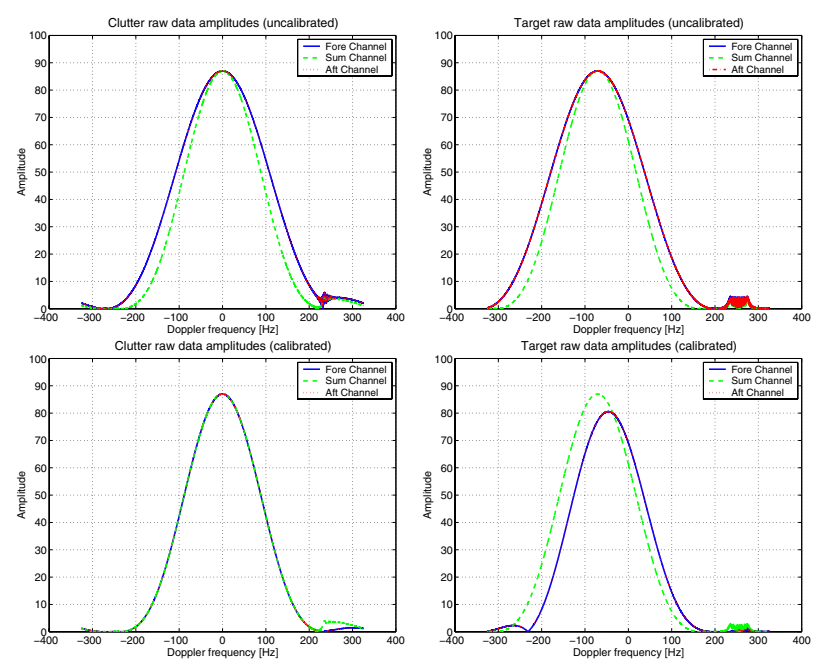

Fig. 1. SAR raw data signals of corresponding clutter and target $\left(v_{y}=\right.$ $2 \mathrm{~m} / \mathrm{s}, S C R=0 d B$ ), before and after calibration to the clutter signals. 


\section{Target Motion Parameter Estimation with DPCA-ATI VERSUS ATI}

The ATI signal is the sum of the target signal with power $S$ and phase $\theta$ and the corresponding clutter signal with power $C$ and zero ATI phase. The angle difference $\alpha$ between the target signal ATI phase $\theta$ and the sum signal ATI phase can be derived by application of the cosine rule and results in:

$$
\alpha(\theta, S C R)=\operatorname{acos}\left(\frac{S C R+\cos (\theta)}{\sqrt{1+S C R^{2}+2 \cdot S C R \cdot \cos (\theta)}}\right) .
$$

The ATI phase bias $\alpha$ versus SCR and target velocity is shown in figure 2. The phase bias is low for SCR larger than $10 \mathrm{~dB}$. For SCR smaller than $0 \mathrm{~dB}$ the ATI phase is strongly biased such that any target velocity results in an ATI phase close to the clutter ATI phase.

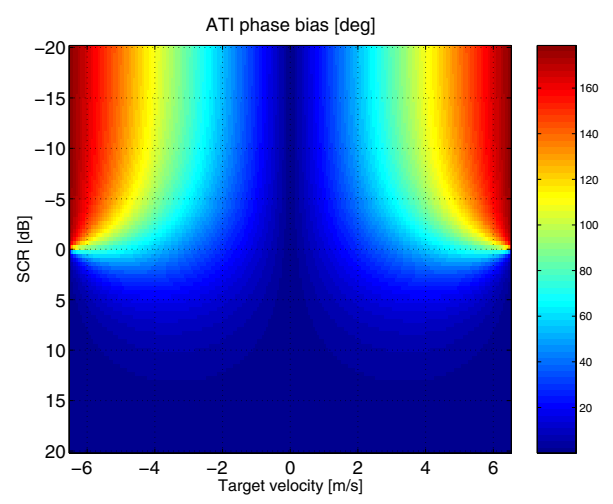

Fig. 2. ATI phase bias versus target across-track velocity and SCR.

\section{A. Simulations}

In figure 3 a simulation result for the Canadian airborne experimental two-channel radar system $C V 580$ is shown where a moving target with radial velocity $v_{y}=3 \mathrm{~m} / \mathrm{s}$ and its corresponding clutter signal are overlaid and the azimuth focussing is matched to the target velocity. The top left image shows the unbiased target ATI signal of the two-channel system, the bottom left image shows the clutter plus target ATI signal when $S C R=0 \mathrm{~dB}$. The clutter interference causes an ATI phase bias of $41^{\circ}$ as was expected from (17). The top right image shows the target DPCA-ATI signal, which is generated by use of the sum channel as the third channel. The expected target phase is half the phase of the ATI signal. The same phase is also obtained in the target plus clutter case for the DPCA-ATI processing as can be seen in the bottom right image.

\section{B. Real Data Results}

Real data collected by the Canadian airborne experimental radar system $C V 580$ in November 2000 also provide a good example of the advantages of the synthetic antenna diversity technique. A target that has about a $10 \log _{10}(6)=7.8 \mathrm{~dB}$
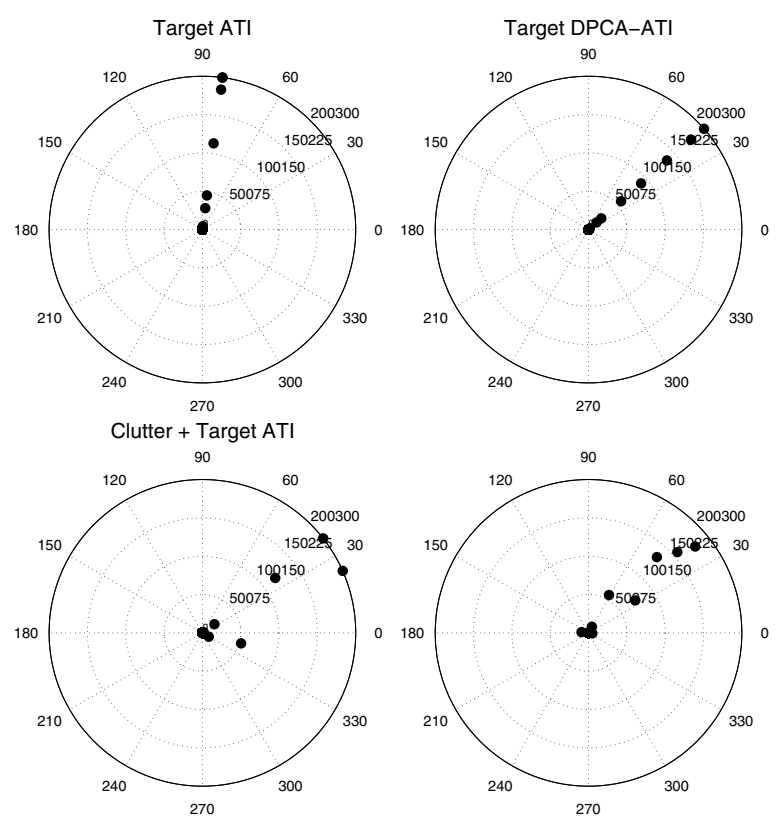

Fig. 3. ATI versus DPCA-ATI velocity estimation $\left(v_{y}=3 \mathrm{~m} / \mathrm{s}, S C R=\right.$ $0 d B$, azimuth compression matched to moving target). Expected target ATI phase: $\theta_{A T I}=82^{\circ}$, expected target DPCA-ATI phase: $\theta_{D P C A-A T I}=$ $41^{\circ}$.

SCR is illustrated in an ATI polar plot in figure 4 on the left. The corresponding DPCA-ATI polar plot is seen on the right. The phase of the DPCA-ATI signal should be half the phase of the unbiased ATI signal. The figures show that this is approximately so. In the right image the absence of clutter can clearly be seen. Careful examination reveals that the DPCAATI signal should yield a higher velocity estimate than the ATI phase. For the target under examination, with $S C R=7.8 \mathrm{~dB}$, and an estimated ATI phase of $\theta_{\text {bias }}=45^{\circ}$ (from figure 4 on the left) one expects that the true ATI phase should be $52^{\circ}$ and the true DPCA-ATI phase $26^{\circ}$. The DPCA-ATI estimate yields an estimate of $26.4^{\circ}$.

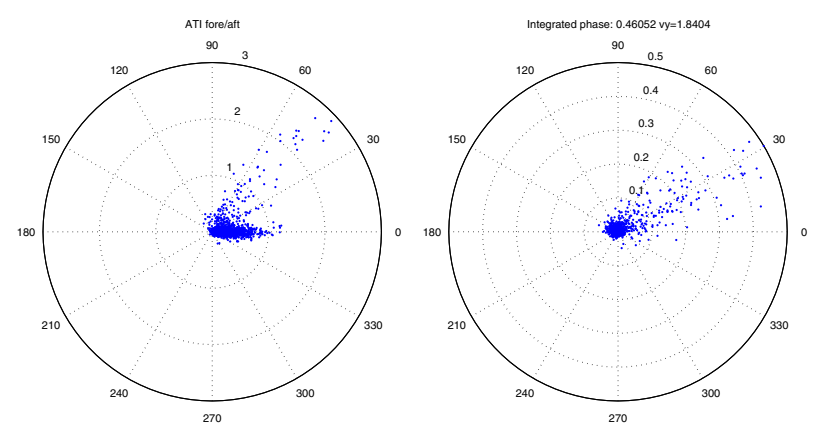

Fig. 4. Real data ATI polar plot (left) and DPCA-ATI polar plot (right).

In figure 5 DPCA-ATI and ATI are compared in an iterative estimation procedure using measured data collected in 1999 by the $C V 580$. The matched filter for azimuth compression in iteration $n+1$ is adapted to the across-track velocity, 
which was estimated in the $n^{t h}$ iteration. In the DPCA-ATI procedure a synthetic channel is generated and DPCA-ATI is accomplished as is described in section III. In the classic ATI procedure the data of the two-channel system are calibrated with the digital channel balancing technique from [6], while in the ATI procedure the two-channel data are calibrated by use of the measured antenna patterns. In the left image the phase measurement was achieved by summing and averaging all data in an area including the target with amplitudes not less than $5 \mathrm{~dB}$ below the maximum target amplitude value. In the right image the phase measurement was achieved by summing and averaging all data in an area including the target with amplitudes not less than $10 \mathrm{~dB}$ below the maximum target amplitude value. In the $10 \mathrm{~dB}$ case, one expects more clutter interference. The results show that the classic ATI technique and the ATI technique using the measured antenna patterns for calibration both yield target velocities that are too low. This is the case even more when the averaging is extended to lower amplitude values as can be seen in the right image. The DPCA-ATI procedure is not affected by the choice of the threshold.
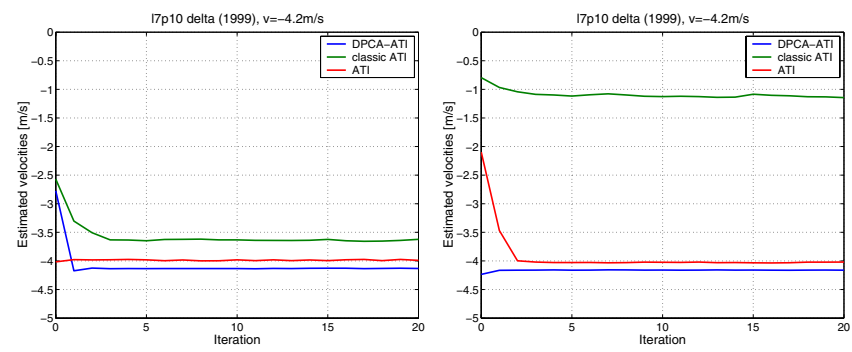

Fig. 5. Iterative estimation of target across-track velocity $\left(v_{y}=-4.2 \mathrm{~m} / \mathrm{s}\right)$, where the azimuth matched filter is adapted to the target across-track velocity. Left: threshold $-5 \mathrm{~dB}$ below maximum. Right: threshold $-10 \mathrm{~dB}$ below maximum.

In figure 6 a low SCR example from real data collected by the $C V 580$ in 2000 is shown, where in the top left ATI image the target signal cannot be identified. The target SCR is low in this case because the target matched filter is not matched in Doppler centroid to the moving target - it is matched to the stationary clutter. The signal can be identified in the DPCA image on the right. On the bottom the iterative estimation procedures are shown. While the classic ATI and the ATI procedure using the measured antenna patterns for calibration yield velocity estimates close to zero velocity, the DPCA-ATI procedure achieves the correct velocity estimate. Due to the high clutter impact the ATI performance is no more influenced by the threshold as can be seen from a comparison of the bottom left and right images.

\section{SUMMARY}

This paper suggests a new mode for processing two-channel SAR data in order to estimate the velocity of moving targets. A third phase center is generated by summation of the fore and aft channel data, which allows then DPCA-ATI processing. However, the synthetic channel manifests not only a third

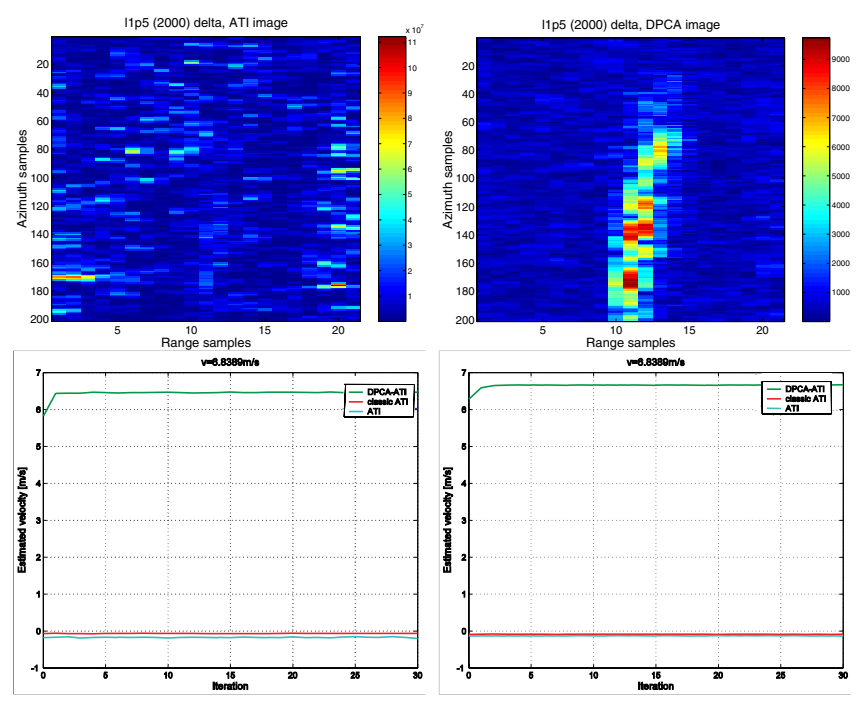

Fig. 6. Top left: absolute values of ATI image. Top right: absolute values of DPCA image. Bottom: Iterative estimation of target across-track velocity $\left(v_{y}=6.8 \mathrm{~m} / \mathrm{s}\right)$, where the azimuth matched filter is adapted to the target across-track velocity. Left: threshold $-3 \mathrm{~dB}$ below maximum. Right: threshold $-10 \mathrm{~dB}$ below maximum.

phase center which allows the clutter suppression, but also a different antenna pattern weighting. Calibration must properly account for the modified antenna pattern though some phase spreading of the DPCA-ATI signal is inevitable.

The mode is compared to ATI, a classical method for estimating the moving target velocity in two-channel SARGMTI. It is shown, conclusively, via simulations and analysis of measured data that a clutter free velocity estimate can be made which offers an improvement over the clutter biased estimate provided by classical ATI.

\section{ACKNOWLEDGEMENTS}

The authors would like to thank Dr. Chuck Livingstone and DRDC for providing the data used in the analysis.

\section{REFERENCES}

[1] S. Chiu, C. Gierull, Multi-Channel Receiver Concepts for RADARSAT-2 Ground Moving Target Indication, EUSAR, Dresden (Germany), May 2006

[2] J.H.G. Ender, Space-time processing for multichannel synthetic aperture radar, Electronics \& Communication Engineering Journal, February 1999

[3] J.H.G. Ender, The Airborne Multi-Channel SAR System AER-II, EUSAR, Koenigswinter, Germany, 1996

[4] C.H. Gierull, Moving Target Detection with Along-Track SAR Interferometry - A Theoretical Analysis, Defense Research Establishment Ottawa (DREO), Aerospace Radar \& Navigation Section, August 2002

[5] C.H. Gierull, Statistics of SAR Interferograms with Application to Moving Target Detection, Defense Research Establishment Ottawa (DREO), Aerospace Radar \& Navigation Section, March 2002

[6] C.H. Gierull, Digital Channel Balancing of Along-Track Interferometric SAR Data, Defense Research Establishment Ottawa (DREO), Aerospace Radar \& Navigation Section, March 2003

[7] R. Klemm, Principles of space-time adaptive processing, IEE Radar, Sonar, Navigation and Avionics Series 12, The Institution of Electrical Engineers, London, United Kingdom, 2002

[8] R.K. Raney, H. Runge, R. Bamler, I.G. Cumming, and F.H. Wong, Precision SAR Processing Using Chirp Scaling, IEEE Transactions on Geoscience and Remote Sensing, vol. 32, No. 4, July 1994 